\title{
ON THE SOLUTION OF CERTAIN \\ DUAL INTEGRAL EQUATIONS
}

\author{
by J. BURLAK
}

(Received 4th February 1962)

1. In this note we consider the formal solution of the dual integral equations

$$
\begin{gathered}
\int_{0}^{\infty} u^{\alpha} \psi(u) J_{v}(x u) d u=f(x) \quad(0 \leqq x<1), \\
\int_{0}^{\infty} \psi(u) J_{v}(x u) d u=g(x) \quad(x>1),
\end{gathered}
$$

where $f(x)$ and $g(x)$ are given and $\psi(x)$ is to be found. The direct solution of these equations has been given by Noble [1] but we shall show that they may be solved more easily if they are first reduced to a form in which $g(x) \equiv 0$.

Thus, following a suggestion of Gordon [2], we put

$$
\psi(x)=\psi_{1}(x)+\psi_{2}(x)
$$

where $\psi_{2}(x)$ is chosen to satisfy the equations

$$
\begin{aligned}
& \int_{0}^{\infty} \psi_{2}(u) J_{v}(x u) d u=0 \quad(0 \leqq x<1), \\
& \int_{0}^{\infty} \psi_{2}(u) J_{v}(x u) d u=g(x) \quad(x>1) .
\end{aligned}
$$

Then we find that $\psi_{1}(x)$ is the solution of the dual integral equations

$$
\begin{gathered}
\int_{0}^{\infty} u^{\alpha} \psi_{1}(u) J_{v}(x u) d u=h(x) \quad(0 \leqq x<1), \\
\int_{0}^{\infty} \psi_{1}(u) J_{v}(x u) d u=0 \quad(x>1),
\end{gathered}
$$

where

$$
h(x)=f(x)-\int_{0}^{\infty} u^{\alpha} \psi_{2}(u) J_{v}(x u) d u
$$

and, by the Hankel inversion theorem (see Sneddon [3]) applied to (4) and (5),

$$
\psi_{2}(x)=x \int_{1}^{\infty} u g(u) J_{v}(x u) d u .
$$

Now the solution of equations of the form (6) and (7) has been given by several authors: 
Titchmarsh [4] (for the case $0<\alpha<2$ ), Busbridge [5] (for the case $-2<\alpha<0$ ), Gordon [2] and Copson [6] (for both cases). Thus, all that remains is to reduce the resulting solution of (6), (7), (8) and (9) to its simplest form.

The method of doing this, described below, is similar in some respects to that used by Noble [7] in treating the case $f \equiv 0$ (he obtained the solution of (1) and (2) by superposing the solutions in the two cases $f \equiv 0$ and $g \equiv 0$ ). He used the Busbridge form [5] of the solution of (6) and (7), which complicates the analysis, and his argument differs in many details from that given below. However, the main disadvantage of his method is that it applies only to the case $-2<\alpha<0$ : various devices (integration by parts or transformation of the original dual integral equations) are needed before the case $0<\alpha<2$ can be treated.

We show below that the argument leading to the form of the solution is identical in the cases $0<\alpha<2$ and $-2<\alpha<0$. It is only in establishing the least restrictive conditions on $v$ that we need some minor modifications in the argument in the case $-2<\alpha<0$. Further, the use of Copson's form [6] of solution (Copson's method for the case $g \equiv 0$ copes equally well with the cases $-2<\alpha<0$ and $0<\alpha<2$ ) not only simplifies the analysis but also yields the final solution in a symmetrical form which shows that there is no essential difference between the cases $f \equiv 0$ and $g \equiv 0$. This is confirmed by the work of Lowengrub and Sneddon [8] who showed that Copson's method applies equally to the case $f \equiv 0$. The same is true of Gordon's method [2] (see [9]).

We may remark at this point that the conditions on $v$ are not always stated explicitly. As they are not the same for all methods of solution we summarize them here. In the case $-2<\alpha<0$, the condition $2 v+\alpha+2>0$ was required in [6], [7], [8], [9] and is also required below. In [1] Noble required $v>0$, which is unnecessarily restrictive. In the case $0<\alpha<2$, Busbridge [5] required $2 v-\alpha+3>0$ and $2 v+\alpha+1>0$; the condition $2 v+\alpha>0$ was imposed in [6], [8], [9] and below: it is more restrictive than the Busbridge condition for $0<\alpha<\frac{3}{8}$, less restrictive for $\frac{3}{2}<\alpha<2$. In [1] Noble required the even more restrictive condition $2 v-\alpha+2>0$ and $2 v+\alpha>0$. We note finally that Gordon [2] required $v>-1$ in both cases -the least restrictive condition of all. However this advantage appears to be lost when his method is adapted to the case $f \equiv 0$ (see [9]). It would be of interest if a definitive set of conditions could be found.

2. We now simplify the solution of (6) and (7), treating the cases $0<\alpha<2$ and $-2<\alpha<0$ together as far as possible.

Copson's form of the solution is [6]

$$
\psi_{1}(x)=\frac{(2 x)^{1-\frac{1}{b} \alpha}}{2 \Gamma\left(1+\frac{1}{2} \alpha\right)} \int_{0}^{1} y^{-\nu-\frac{1}{2} \alpha} J_{v+\frac{1}{\alpha} \alpha}(x y) \frac{d}{d y}\left\{\int_{0}^{y} u^{\nu+1} h(u)\left(y^{2}-u^{2}\right)^{\frac{1 \alpha}{2}} d u\right\} d y,
$$

which is valid for $0<\alpha<2,2 v+\alpha>0$ and for $-2<\alpha<0,2 v+\alpha+2>0$. (Copson gives the second condition as $-2<\alpha<0, v>-1$, but this is in error.)

Thus, by (3), (8), (9) and (10), the solution of the original equations (1) and (2) is

$$
\psi(x)=F(x)+G(x)
$$

where 


$$
\begin{aligned}
& F(x)=\frac{(2 x)^{1-\frac{1}{\alpha} \alpha}}{2 \Gamma\left(1+\frac{1}{2} \alpha\right)} \int_{0}^{1} y^{-v-\frac{1}{2} \alpha} J_{v+\frac{1}{\alpha} \alpha}(x y) \frac{d}{d y}\left\{\int_{0}^{y} u^{v+1} f(u)\left(y^{2}-u^{2}\right)^{\natural \alpha} d u\right\} d y, \\
& G(x)=x \int_{1}^{\infty} u g(u) J_{v}(x u) d u+H(x), \\
& H(x)=-\frac{(2 x)^{1-1 \alpha}}{2 \Gamma\left(1+\frac{1}{2} \alpha\right)} \int_{0}^{1} y^{-\nu-\frac{1}{1} \alpha} J_{\nu+\frac{1}{\alpha} \alpha}(x y) \frac{d}{d y}\left[\int_{0}^{y} u^{\nu+1}\left(y^{2}-u^{2}\right)\right. \\
& \left.\times\left\{\int_{0}^{\infty} v^{\alpha} J_{v}(u v)\left(v \int_{1}^{\infty} \operatorname{tg}(t) J_{v}(v t) d t\right) d v\right\} d u\right] d y .
\end{aligned}
$$

Our aim is to reduce the four-fold integral in (14) to a double integral.

By interchanging the order of integration in the inner three-fold integral in (14) (as our analysis is formal we do not attempt to justify such an interchange) we find that

$$
\begin{aligned}
& I(x)=\int_{0}^{y} u^{v+1}\left(y^{2}-u^{2}\right)^{v^{\alpha}}\left\{\int_{0}^{\infty} v^{\alpha} J_{v}(u v)\left(v \int_{1}^{\infty} t g(t)^{y} J_{v}(v t) d t\right) d v\right\} d u \\
& =\int_{0}^{\infty} v^{\alpha+1}\left\{\int_{1}^{\infty} t g(t) J_{v}(v t)\left(\int_{0}^{y} u^{v+1}\left(y^{2}-u^{2}\right)^{\xi \alpha}(u v) d u\right) d t\right\} d v .
\end{aligned}
$$

But now, by Sonine's first integral (Watson [10, p. 373]),

$$
J_{\mu+v+1}(z)=\frac{z^{\nu+1}}{2^{\nu} \Gamma(v+1)} \int_{0}^{\pi / 2} J_{\mu}(z \sin \theta) \sin ^{\mu+1} \theta \cos ^{2 v+1} \theta d \theta,
$$

which is valid if $\operatorname{Re}(\mu)>-1$ and $\operatorname{Re}(v)>-1$, we see that

$$
\int_{0}^{y} u^{v+1}\left(y^{2}-u^{2}\right)^{\ddagger \alpha} J_{v}(u v) d u=2^{1 \alpha \alpha} \Gamma\left(1+\frac{1}{2} \alpha\right) y^{\nu+k \alpha+1} v^{-1-\frac{1}{2} \alpha} J_{v+\frac{1}{2} \alpha+1}(y v),
$$

provided that $\alpha>-2, v>-1$, where we have replaced $\mu, v, z$ and $\sin \theta$ in (16) by $v, \frac{1}{2} \alpha, y v$ and $u / y$, respectively. The conditions on $\alpha$ and $v$ are satisfied if $0<\alpha<2,2 v+\alpha>0$ and if $-2<\alpha<0,2 v+\alpha+2>0$.

Thus, by (14); (15) and (17),

$$
H(x)=-x^{1-\frac{1}{\alpha} \alpha} \int_{0}^{1} y J_{v+\frac{t a}{2 \alpha}}(x y)\left\{\int_{0}^{\infty} v^{1+1 \alpha \alpha} J_{v+t_{\alpha}}(y v)\left(\int_{1}^{\infty} t g(t) J_{v}(v t) d t\right) d v\right\} d y,
$$

where we have applied the result (Watson [10, p. 18])

$$
\frac{d}{d x}\left\{x^{n} J_{n}(x y)\right\}=y x^{n} J_{n-1}(x y)
$$

to the product $y^{\nu+1 \alpha+1} J_{v+t a+1}(y v)$. If we now use the similar result (Watson [10, p. 18])

$$
\frac{d}{d x}\left\{x^{-n} J_{n}(x y\}=-y x^{-n} J_{n+1}(x y)\right.
$$


to write

$$
y v J_{v+\frac{1}{2} \alpha}(v y)=y^{\nu+\hbar \alpha} v y^{-\nu-j \alpha+1} J_{v+\frac{1}{2} \alpha}(v y)=-y^{\nu+\frac{1}{2} \alpha} \frac{d}{d y}\left\{y^{-\nu-1 \alpha+1} J_{v+\frac{1}{2} \alpha-1}(v y)\right\}
$$

and again interchange the order of integration in (18), we find that

$$
H(x)=x^{1-\frac{1}{2} \alpha} \int_{0}^{1} y^{\nu+1 \alpha} J_{v+\frac{1}{2} \alpha}(x y) \frac{d}{d y}\left\{y^{-v-1 \alpha+1} \int_{1}^{\infty} \operatorname{tg}(t)\left(\int_{0}^{\infty} v^{\frac{1 \alpha}{\alpha}} J_{v}(v t) J_{v+\frac{1}{2} \alpha-1}(v y) d v\right) d t\right\} d y .
$$

The inner integral can be evaluated in terms of the Weber-Schaftheitlin discontinuous integral (Watson [10, p. 398])

$$
\begin{aligned}
& \int_{0}^{\infty} J_{\mu}(x t) J_{v}(y t) t^{-\lambda} d t= \\
& \frac{y^{\nu} \Gamma\left\{\frac{1}{2}(\mu+v-\lambda+1)\right\}}{2^{\lambda} x^{\nu-\lambda+1} \Gamma(v+1) \Gamma\left\{\frac{1}{2}(\lambda+\mu-v+1)\right\}}{ }_{2} F_{1}\left\{\frac{1}{2}(\mu+v-\lambda+1), \frac{1}{2}(v-\lambda+\mu+1) ; v+1 ; y^{2} / x^{2}\right\},
\end{aligned}
$$

where $0<y<x$; a similar expression, with $x$ and $y$, and $\mu$ and $v$, interchanged, holds if $0<x<y$. The integral is convergent if

$$
\operatorname{Re}(\mu+v+1)>\operatorname{Re}(\lambda)>-1 .
$$

Thus, since $y \leqq t$, if we replace $\mu, v$ and $\lambda$ in (22) by $v, v+\frac{1}{2} \alpha-1$ and $-\frac{1}{2} \alpha$, respectively, and use the result

$$
{ }_{2} F_{1}\left(v+\frac{1}{2} \alpha, \frac{1}{2} \alpha ; v+\frac{1}{2} \alpha ; y^{2} / x^{2}\right)=x^{\alpha}\left(x^{2}-y^{2}\right)^{-\frac{1}{2} \alpha} \quad(0<y<x),
$$

we obtain

$$
\int_{0}^{\infty} v^{\frac{1}{\alpha} \alpha} J_{\nu}(v t) J_{v+\frac{1}{2} \alpha-1}(v y) d v=2^{\frac{1}{1} \alpha}\left\{\Gamma\left(1-\frac{1}{2} \alpha\right)\right\}^{-1} y^{v+1 \alpha-1} t^{-v}\left(t^{2}-y^{2}\right)^{-1 \alpha}
$$

provided that $2 v+\frac{1}{2} \alpha>-\frac{1}{2} \alpha>-1$, which is so if $0<\alpha<2,2 v+\alpha>0$ and if $-2<\alpha<0$, $2 v+\alpha>0$. Hence, by (21) and (25),

$$
H(x)=\frac{2^{\frac{1}{\jmath} \alpha} x^{1-\frac{1}{d} \alpha}}{\Gamma\left(1-\frac{1}{2} \alpha\right)} \int_{0}^{1} y^{y+\frac{t}{d} \alpha} J_{v+\frac{1}{2} \alpha}(x y) \frac{d}{d y}\left\{\int_{1}^{\infty} t^{1-y} g(t)\left(t^{2}-y^{2}\right)^{-\frac{1}{t} \alpha} d t\right\} d y .
$$

We now rewrite the double integral in (26):

$$
\int_{0}^{1} \ldots\left\{\int_{1}^{\infty} \ldots d t\right\} d y=\int_{0}^{\infty} \ldots\left\{\int_{y}^{\infty} \ldots d t\right\} d y-\int_{1}^{\infty} \ldots\left\{\int_{y}^{\infty} \ldots d t\right\} d y-\int_{0}^{1} \ldots\left\{\int_{y}^{1} \ldots d t\right\} d y
$$

and, in the first and third integrals on the right, we integrate by parts (using (19)), interchange the order of integration and combine the resulting integrals. This yields 


$$
\begin{aligned}
& H(x)=-\frac{2^{\dagger \alpha} x^{1-\downarrow \alpha}}{\Gamma\left(1-\frac{1}{2} \alpha\right)} \int_{1}^{\infty} y^{\nu+\frac{1}{2} \alpha} J_{v+\frac{1}{\alpha} \alpha}(x y) \frac{d}{d y}\left\{\int_{y}^{\infty} t^{1-v} g(t)\left(t^{2}-y^{2}\right)^{-\frac{1}{\alpha} \alpha} d t\right\} d y \\
& -\frac{2^{1 \alpha} x^{2-1 \alpha}}{\Gamma\left(1-\frac{1}{2} \alpha\right)} \int_{1}^{\infty} t^{1-v} g(t)\left\{\int_{0}^{t} y^{v+1 \alpha \alpha}\left(t^{2}-y^{2}\right)^{-t \alpha} J_{v+\frac{1}{2} \alpha-1}(x y) d y\right\} d t .
\end{aligned}
$$

The second integral in (27) can be simplified by use of a modified form of (17):

$$
\int_{0}^{t} y^{v+\frac{t}{2} \alpha}\left(t^{2}-y^{2}\right)^{-\frac{1}{2} \alpha} J_{v+t \alpha-1}(x y) d y=2^{-\frac{1}{2} \alpha} \Gamma\left(1-\frac{1}{2} \alpha\right) t^{\nu} x^{-1-\frac{1}{2} \alpha} J_{v}(x t),
$$

provided that $-\frac{1}{2} \alpha>-1, v+\frac{1}{2} \alpha-1>-1$, which is so if $0<\alpha<2,2 v+\alpha>0$ and if $-2<\alpha<0,2 v+\alpha>0$. Hence, by (13), (27) and (28),

$$
G(x)=-\frac{2^{1 \alpha} x^{1-\frac{1}{2} \alpha}}{\Gamma\left(1-\frac{1}{2} \alpha\right)} \int_{1}^{\infty} y^{\nu+1 \alpha} J_{v+\frac{1}{2} \alpha}(x y) \frac{d}{d y}\left\{\int_{y}^{\infty} t^{1-\nu} g(t)\left(t^{2}-y^{2}\right)^{-1 \alpha} d t\right\} d y
$$

provided that $0<\alpha<2,2 v+\alpha>0$ or $-2<\alpha<0,2 v+\alpha>0$, and the solution of (1) and (2) is given by (11), (12) and (29).

3. The case $-2<\alpha<0$. As we have seen, the above argument holds for $0<\alpha<2$, $2 v+\alpha>0$ and for $-2<\alpha<0,2 v+\alpha>0$. To relax the restriction on $v$ in the second case we follow the above argument as far as (18), which holds when $-2<\alpha<0,2 v+\alpha+2>0$ and which can be written

$$
H(x)=-x^{1-t \alpha} \int_{0}^{1} y J_{v+\frac{1}{2} a}(x y)\left\{\int_{1}^{\infty} t g(t)\left[\int_{0}^{\infty} v^{1+\frac{1}{\alpha} \alpha} J_{v}(v t) J_{v+\frac{1}{\alpha} \alpha}(v y) d v\right] d t\right\} d y .
$$

The inner integral can be evaluated by a modified form of (25):

$$
\int_{0}^{\infty} v^{1+\frac{1}{\alpha}} J_{v}(v t) J_{\nu+t \alpha}(v y) d v=2^{1+\frac{1}{d} \alpha}\left\{\Gamma\left(-\frac{1}{2} \alpha\right)\right\}^{-1} y^{\nu+t \alpha} t^{-v}\left(t^{2}-y^{2}\right)^{-1-\frac{t}{2} \alpha},
$$

provided that $2 v+\frac{1}{2} \alpha+1>-1-\frac{1}{2} \alpha>-1$, which is so if $-2<\alpha<0,2 v+\alpha+2>0$. Thus (30) becomes

$$
H(x)=-\frac{2^{1+1 \alpha \alpha} x^{1-\frac{1}{1} \alpha}}{\Gamma\left(-\frac{1}{2} \alpha\right)} \int_{0}^{1} y^{v+\frac{1}{2} \alpha+1} J_{v+\frac{1}{2} \alpha}(x y)\left\{\int_{1}^{\infty} t^{1-v} g(t)\left(t^{2}-y^{2}\right)^{-1-\frac{1}{\alpha} \alpha} d t\right\} d y .
$$

We now use the same argument as led from (26) to (27) (omitting the integrations by parts) to obtain

$$
\begin{gathered}
H(x)=\frac{2^{1+1 \alpha} x^{1-1 \alpha}}{\Gamma\left(-\frac{1}{2} \alpha\right)} \int_{1}^{\infty} y^{\nu+1 \alpha+1} J_{v+\frac{1}{2} \alpha}(x y)\left\{\int_{y}^{\infty} t^{1-\nu} g(t)\left(t^{2}-y^{2}\right)^{-1-1 \alpha} d t\right\} d y \\
-\frac{2^{1+1 \alpha} x^{1-1} \alpha}{\Gamma\left(-\frac{1}{2} \alpha\right)} \int_{1}^{\infty} t^{1-v} g(t)\left\{\int_{0}^{t} y^{v+1 \alpha+1}\left(t^{2}-y^{2}\right)^{-1-1 \alpha} J_{v+\frac{1}{2} \alpha}(x y) d y\right\} d t .
\end{gathered}
$$


The rest of the argument follows as above, if we use a second modified form of (17):

$$
\int_{0}^{t} y^{\nu+1 \alpha+1}\left(t^{2}-y^{2}\right)^{-1-\frac{1}{d} \alpha} J_{v+\frac{1}{2} \alpha}(x y) d y=2^{-1-t \alpha} \Gamma\left(-\frac{1}{2} \alpha\right) t^{\nu} x^{\frac{1}{1} \alpha} J_{v}(x t),
$$

provided that $v+\frac{1}{2} \alpha>-1,-1-\frac{1}{2} \alpha>-1$, which is so if $-2<\alpha<0,2 v+\alpha+2>0$. If we then write

$$
\left(t^{2}-y^{2}\right)^{-1-\frac{1}{d} \alpha}=\left\{(-2 y)\left(-\frac{1}{2} \alpha\right)\right\}^{-1} \frac{d}{d y}\left(t^{2}-y^{2}\right)^{-1 \alpha},
$$

we obtain precisely (29), under the conditions $-2<\alpha<0,2 v+\alpha+2>0$. This completes the solution in the two cases $0<\alpha<2,2 v+\alpha>0$ and $-2<\alpha<0,2 v+\alpha+2>0$.

\section{REFERENCES}

1. B. Noble, Certain dual integral equations, J. Math. and Phys. 37 (1958), 128-136.

2. A. N. Gordon, Dual integral equations, J. London Math. Soc. 29 (1954), 360-363.

3. I. N. Sneddon, Fourier transforms (McGraw-Hill, New York, 1951), p. 52.

4. E. C. Titchmarsh, Introduction to the theory of Fourier integrals (Clarendon Press, Oxford, 1937), pp. 334-339.

5. I. W. Busbridge, Dual integral equations, Proc. London Math. Soc. (2) 44 (1938), 115-129.

6. E. T. Copson, On certain dual integral equations, Proc. Glasgow Math. Assoc. 5 (1961), 21-24.

7. B. Noble, On some dual integral equations, Quart. J. Math. Oxford ser. (2) 6 (1955), 81-87.

8. M. Lowengrub and I. N. Sneddon, On certain dual integral equations, Air Force Technical Report, Contract No. AF 49(638)-892, 1961 (to be published shortly).

9. J. Burlak, On Gordon's method of solving dual integral equations (to appear shortly).

10. G. N. Watson, A treatise on the theory of Bessel functions (University Press, Cambridge, 2nd edition, 1944).

North Carolina State College

Raleigh, North Carolina, U.S.A.

(Permanent Address: The University, Glasgow, W. 2.) 